\title{
A PROGRESS REPORT ON THE ESTABLISHMENT OF THE RADIO / OPTICAL REFERENCE FRAME
}

\author{
J. L. Russell AND K. J. Johnston \\ U. S. Naval Research Lab, Code 4030 \\ Washington, D.C. 20375 USA \\ C. DE Vegt AND N. Zacharias \\ Hamburger Sternwarte, Gojenbergsweg 112 \\ D-2050 Hamburg 60 \\ Germany, Fed. Republic \\ C. MA AND D. ShafFer \\ Crustal Dynamics Project \\ Goddard Space Flight Center, Code 621.9 \\ Greenbelt, MD 20771 USA
}

D.L. JAUNCEY AND G.L. WhITE
Australia Telescope National Facility
CSIRO Division of Radiophysics
Sydney, Australia

G. Nicolson and A. Kemball

Hartebeesthoek Radio Astronomy Observatory

Meiring Naude Rd

Pretoria, South Africa

R. HiNdSLEY AND J. Hughes

U. S. Naval Observatory

Washington, D.C. 20392

\author{
J.E. REYNOLDS \\ Mount Stromlo and Siding Spring Obs. \\ Australian National University \\ Woden, ACT 2606, Australia
}

\section{Introduction}

In 1987 we began a 5-year program to establish a reference system of at least 400 extragalactic sources which are compact and flat spectrum in the radio and which also display optical emission. This reference frame is to be global with about one source per $100 \mathrm{sq}$ deg (Johnston, et al., 1988). The program incorporates some data which had been obtained previously for other purposes. Altogether, in one or another aspect of the program, 489 sources have been considered so far.

This progress report summarizes the program as of September 1989. The published radio and optical positions are in Ma, et al. (1990) and Russell, et al. (1990a,b). 


\section{Radio Observations}

To date we have obtained S/X dual frequency VLBI observations of 347 sources, 325 of which have at least one good observation and about 300 of which are suitable for inclusion in the radio reference frame. About two-thirds of these are in the northern hemisphere.

The distribution of observed radio sources is shown in Figure 1. The symbols in the figure indicate whether the sources were first observed as part of the NASA Crustal Dynamics Project (source list from GLOBL solution GBL353; various baselines), the new northern hemisphere observations (GBL475; Fairbanks-Hatcreek-Maryland Point), the mid-southem observations (GBL576; KauaiKashima-Tidbinbilla), or the far southern observations (GBL476; Tidbinbilla-Hartebeesthoek). The largest gaps in the distribution are in the galactic plane. The sources observed to date include 198 of the 233 recommended by the IAU working group on the Radio/Optical Reference Frame (Argue, et al, 1984). The positions for the southern sources in general are not as accurate as the northem because of differences in the number of observations; the CDP sources are especially well-observed and accurate.

The radio results are adopted as the positions of the sources and establish the reference frame. More details of the formation of the radio reference frame from VLBI are given by Ma (1990) and Ma et al. (1990). We note that the RA zero point is fixed using positions of optical counterparts of 28 quasars which have been derived on the system of the FK5.

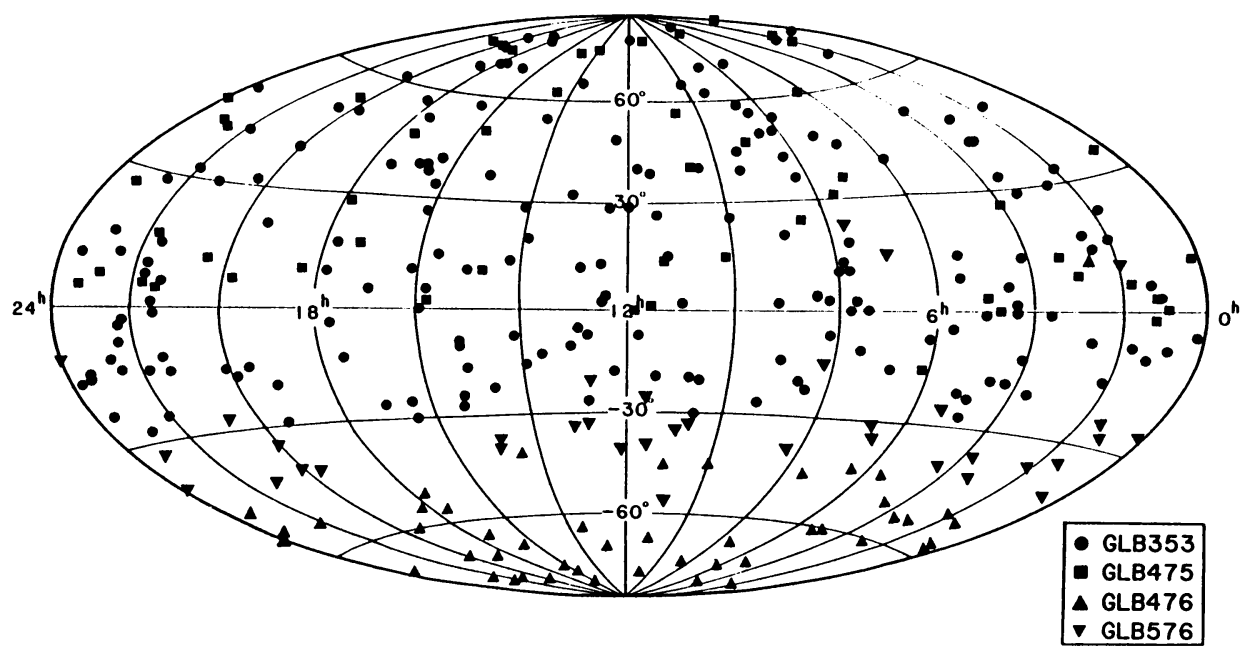

Figure 1. Distribution of sources which have been observed with VLBI. 


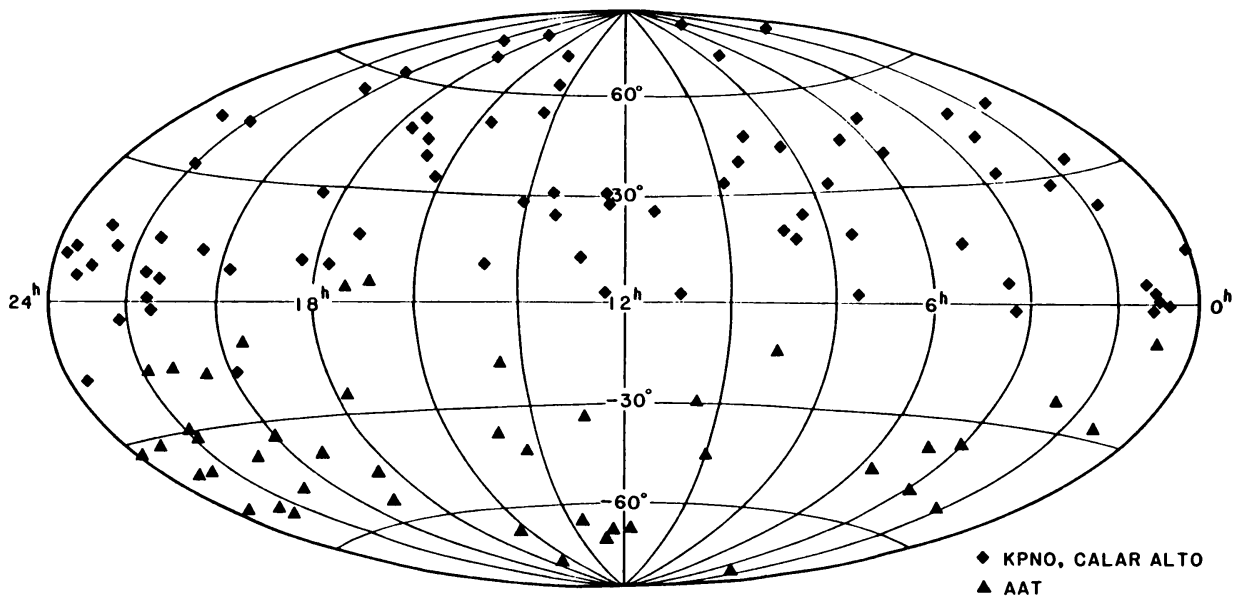

Figure 2. Distribution of sources which have source plates.

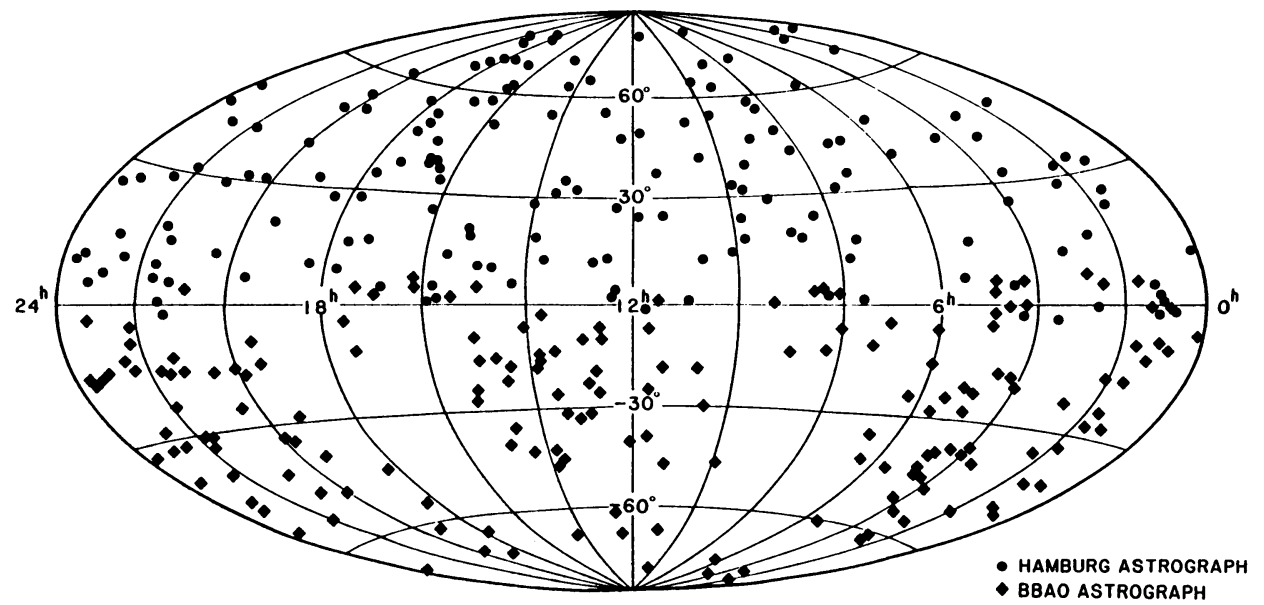

Figure 3. Distribution of sources which have astrograph plates. 


\section{Optical Observations}

The optical positions of the radio/optical sources are obtained with a two-step procedure. The position of the optical counterpart, generally $18<\mathrm{m}_{v}<22$, is determined using deep prime focus photographs or CCD images from 4-m class telescopes, so far the KPNO 4-m, the Calar Alto telescope and the AAT. The position is referred to secondary reference stars of intermediate magnitude $\left(12<\mathrm{m}_{\mathrm{v}}<16\right)$ which can be measured relative to AGK3RN (Corbin, 1979) or SRS (Smith and Jackson, 1985) stars using plates from wide field astrographs, specifically those of Hamburger Sternwarte in Hamburg, FRG and Black Birch Astronomical Observatory, the USNO station in New Zealand.

As of September 1989, at least four astrograph plates each have been obtained for 170 northern sources and at least one plate each has been obtained for 173 southem sources. We have source plates of only a fraction of those, 126 total, because of the difficulty of obtaining observing time on the large instruments. Figure 2 shows the distribution of sources for which we have obtained at least one source plate; Figure 3 shows the distribution of sources with astrograph plates.

\section{Summary}

We plan to reobserve the sources annually with VLBI to improve the radio reference system and to continue acquisition of optical data. We will also be observing new sources to increase the density of the source distribution in the reference frame and to replace some of the marginal sources currently included.

\section{References}

Argue, A.N., de Vegt, C., Elsmore, B., Fanselow, J., Harrington, R., Hemenway, P., Johnston, K.J., Kuehr, H., Kumkova, I., Neill, A.E., Walter, H., and Witzel, A. (1984) Astron. Astrophys., 130, 191-199.

Corbin, T. (1979) in "Modern Astrometry," IAU Colloq. 48, F.V. Prochazka and R.H. Tucker (eds.), (Univ. Obs., Vienna), p. 505.

Johnston, K.J., Russell, J., de Vegt, C., Hughes, J., Jauncey, D., White, G., and Nicholson, G. (1988) in "The Impact of VLBI on Astrophysics and Geophysics," IAU Symp. 129, M. Reid and J. Moran (eds.), (D. Reidel, Dordrecht), p. 317.

Ma, C. (1990) this symposium

Ma, C., Shaffer, D.B., de Vegt, Chr., Johnston, K.J., and Russell, J.L. (1990) Astron. J. 99, no. 4. Russell, J.L., Johnston, K.J., Jauncey, D., White, G., Reynolds, J., Harvey, B., Nothnagel, A., Nicholson, G., Kingham, K., Ma, C., de Vegt, C., Hindsley, R., and Zacharias, N. (1990a) in preparation.

Russell, J.L., Johnston, K.J., Ma, C., Shaffer, D., and de Vegt, C. (1990b) in preparation.

Smith, C., Jackson, E., (1985) Celest. Mechanics 37, 277. 